\title{
NUMERICAL HOMOGENIZATION TECHNIQUES FOR THE EVALUATION OF MECHANICAL BEHAVIOR OF A COMPOSITE WITH SMA INCLUSIONS
}

\author{
Valerio Alecci, Silvia Briccoli Bati And Giovanna Ranocchiai
}

\begin{abstract}
Numerical procedures are developed for the homogenization and evaluation of the stress field in a composite as a consequence of the presence of embedded SMA (shape-memory alloy) wires. In particular, the elastic field developed at the end of the SMA wire self-strain process is studied, knowledge of which is necessary to evaluate the feasibility of such a hybrid composite.

First, the numerical procedures are applied to the study of both a representative volume element (RVE) included in a theoretically infinite periodic medium and a RVE located near the medium free boundary, in order to evaluate the tangential stress field generated at the end of the fiber; then they are applied to the study of a plate able to bend after the effect of self-strain of the SMA wire.

Observations are reported about the obtained results and about the similarities and the differences between the two problems.
\end{abstract}

\section{Introduction}

In the early 1990s, as the exciting potential of hybrid composites [Ahmad et al. 1990] came to be understood, researchers investigated a range of problems, from the mechanical aspects of such materials [Boyd and Lagoudas 1994; Barrett and Gross 1996; Aboudi 1997] to their construction, characterization, and production [Wei et al. 1997]. More recently, significant attention has been given to polymeric matrix adaptive composites [Bidaux et al. 1994], cement matrix composites [Zheng et al. 2001], metal matrix (aluminum), and carbon or glass fiber-reinforced matrix composites [Wei et al. 1997]. Particularly, in [Baltá et al. 2002; Xu et al. 2002; Zheng et al. 2002; Murasawa et al. 2004; Tsoi et al. 2004], some specimens of composite material with integrated SMA (shape-memory alloy) wires are presented.

An interesting analysis of the interfacial quality in activated shape-memory alloy composites (CSMA) appeared in [Zheng et al. 2003]. Qualitative aspects were experimentally evaluated by differential scanning calorimeter, and quantitative results were obtained assuming that the behavior of the two phases, fiber and matrix, was linearly elastic. The radial and circumferential stress was calculated from the thermoelasticity solution provided by Hecker et al. [1970] to evaluate the residual stresses in composite cylinders originated during the cooling of the composite from fabrication temperatures.

Recently, works by Marfia and Sacco [2005] and Marfia [2005] were published, concerning micromechanical modeling of SMA-wire-reinforced materials. The authors proposed two homogenization procedures based on the following assumptions: the martensite volume fraction depends on the wire temperature and only on the normal stress acting in the fiber direction, and the inelastic strain occurs along the fiber direction. A finite element model in the two-dimensional setting for SMA hybrid composite plates was proposed by Daghia et al. [2006]. Finally, [Marfia and Sacco 2007] presents a nonlinear

Keywords: numerical homogenization, composite materials, shape-memory alloys. 
laminate finite element able to reproduce both superelasticity and a memory effect for a laminated plate containing one or more ply made of SMA reinforced composite. These authors apply numerical homogenization procedures in order to develop the overall mechanical response of a CSMA, as a step of evolutive multiscale analysis.

The use of homogenization techniques seems quite apt to analyze the problem of the stress field induced by the self-strain of a portion of material, as the basic formulas of homogenization techniques were derived simulating the homogeneous deformation of an ellipsoidal region surrounded by elastic material [Eshelby 1957]. The development of numerical homogenization techniques for periodic media in the 80's [Duvaut 1984; Suquet 1985] produced the analytical solution for homogenization problems in which the shape of the periodic cell is by far dissimilar to ellipsoid, elliptic cylinders, or ribbons. In particular, several applications were developed in order to deduce at first in-plane elastic characteristics of masonry panels, characterized by two-directional periodicity under a plane stress assumption, generalized plane strain, and three-directional modeling [Anthoine 1995; 1997]; recently, out-of-plane characteristics of masonry walls were deduced via numerical homogenization [Cecchi et al. 2005; Milani et al. 2006].

In this paper the development of a numerical procedure for the homogenization and the evaluation of the stress field in a composite as a consequence of the shape-memory effect of embedded SMA wires are described. In particular, the elastic field developed at the end of the self-strain process of the SMA wire is studied, neglecting the intermediate stages. The stress state that is produced in the composite at the final stage is the most severe of the whole load history and knowledge of it is necessary to evaluate the feasibility of such a hybrid composite.

At first, the numerical homogenization procedure is applied to the study of an infinite medium, then to the study of a plate able to bend after the effect of self-strain of the SMA wire.

The paper is organized as follows: Section 2 is devoted to the homogenization procedure and to the evaluation of the elastic field produced by the activation of the SMA wires, for a three-dimensional solid; attention was focused on the boundary condition and on the possible load conditions; the derivation of the null condition for the average stress is reported in the Appendix; the results obtained for a case study are reported. Section 3 is devoted to the homogenization procedure and to the evaluation of the elastic field produced by the activation of the SMA wires embedded in a plate; the results for a case study are reported. Finally, conclusions are reported in Section 4.

\section{Elastic field in a three-dimensional solid}

2.1. Homogenization. The composite material subjected to analysis is a matrix-fiber composite made of resin and aligned SMA wires ( $150 \mu \mathrm{m}$ in diameter) assumed to be in a regular array according with Figure 1. The span between the wires is $0.6 \mathrm{~mm}$, and as a consequence, the volume fraction of the inclusion is about $5 \%$. The two phases are assumed to be isotropic with the following elastic constants: $E=70000 \mathrm{MPa}$ and $v=0.33$ (fiber), and $E=20000 \mathrm{MPa}$ and $v=0.4$ (matrix). The values indicated for the SMA fiber represent the effective properties of a typical Ni-Ti alloy, while the Young's modulus and Poisson's ratio of the matrix might agree with those of a composite matrix made of epoxy resin with glass additives inside.

Homogenization techniques aim at describing the behavior of a heterogeneous material by means of the laws of a homogeneous material that is equivalent in some sense. The problem is not trivial when the 

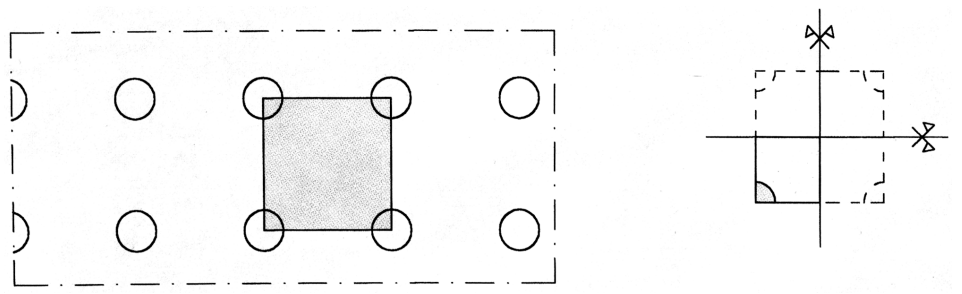

Figure 1. Left: schematic representation of the composite with embedded SMA fibers and localization of the unit cell. The plane shown is perpendicular to the fiber length. Right: region subjected to numerical analysis (continuous line).

parameters to be determined are not additive (extensive) quantities, as in the case of elasticity. Elastic coefficients in fact, like other intensive quantities, cannot be directly averaged in the volume of the body, as they take part in the functional laws among additive quantities.

We wish to produce a macroscopically homogeneous stress field in a heterogeneous body; that is, every representative volume element (RVE) is subjected to the same stress state.

The homogenized elastic tensor at a point $\boldsymbol{x}$ being $T(\boldsymbol{x})=K(\boldsymbol{x}) D(\boldsymbol{x})$, the local constitutive relation the between the stress and strain tensors can be defined as

$$
\langle T\rangle=K^{\text {hom }}\langle D\rangle,
$$

where

$$
\langle T\rangle=\frac{1}{V} \int_{V} T d V, \quad\langle D\rangle=\frac{1}{V} \int_{V} D d V .
$$

To calculate the components of $K^{\text {hom }}$, we must produce an elementary strain state, one of the form

$$
D_{1}=\left[\begin{array}{lll}
1 & 0 & 0 \\
0 & 0 & 0 \\
0 & 0 & 0
\end{array}\right], \quad D_{2}=\left[\begin{array}{lll}
0 & 0 & 0 \\
0 & 1 & 0 \\
0 & 0 & 0
\end{array}\right], \quad \ldots, \quad D_{6}=\left[\begin{array}{lll}
0 & 0 & 0 \\
0 & 0 & 1 \\
0 & 1 & 0
\end{array}\right] .
$$

To do this, Hashin's lemmas are commonly used when dealing with nonperiodic heterogeneous materials, which guarantee that $\langle T\rangle=T$ if $T \boldsymbol{n}=\langle T\rangle \boldsymbol{n}$ on $\partial V$ and $T$ is divergence free, and $\langle D\rangle=D$ if $\boldsymbol{u}=D \boldsymbol{x}$ on $\partial V$.

When dealing with periodic heterogeneous materials the periodic cell represents the RVE; if the cell is sufficiently far from the boundary, stress and strain are periodic fields and periodicity conditions may be assumed on the border of the periodic cell, that is $\boldsymbol{t}=T \boldsymbol{n}$ antiperiodic and $\boldsymbol{u}=\langle D\rangle \boldsymbol{x}+\boldsymbol{u}^{p}$, where $\boldsymbol{u}^{p}$ is periodic, that is assumes the same value on the boundary of the cell.

The last equality can be written

$$
D(\boldsymbol{u})=\langle D\rangle+D\left(\boldsymbol{u}^{p}\right)
$$

or

$u_{1}=d_{11} x_{1}+d_{12} x_{2}+d_{13} x_{3}+u_{1}^{\mathrm{per}}, \quad u_{2}=d_{21} x_{1}+d_{22} x_{2}+d_{23} x_{3}+u_{2}^{\mathrm{per}}, \quad u_{3}=d_{31} x_{1}+d_{32} x_{2}+d_{33} x_{3}+u_{3}^{\mathrm{per}}$.

The field equations of elasticity, neglecting the body forces, are

$$
\operatorname{div}(K(\boldsymbol{x}) D(\boldsymbol{u}))=0
$$


and substituting the expression of displacement from (2-4):

$$
\operatorname{div}\left(K(\boldsymbol{x}) D\left(\boldsymbol{u}^{p}\right)\right)+\operatorname{div}(K(\boldsymbol{x})\langle D\rangle)=0 .
$$

$K(\boldsymbol{x})$ is the local value of the elastic tensor and is constant within each phase and discontinuous on the border between the materials. For this reason the second term of (2-6) takes the form of a body force spread over the interface $\partial S$ between the phases:

$$
\boldsymbol{f}=\left(K_{2}-K_{1}\right)\langle D\rangle \boldsymbol{n} \delta_{\partial S},
$$

where $\boldsymbol{n}$ is the unit vector normal to $\partial S$ from phase 2 to phase 1 [Suquet 1985].

If we solve with FEM analysis the problem of (2-6) assigning $\boldsymbol{f}$ from (2-7) as produced by an elementary homogeneous strain tensor, the homogenized elasticity tensor can be calculated from (2-1), where $\langle D\rangle$ is the imposed elementary strain, $D\left(\boldsymbol{u}^{p}\right)$ is the result of the numerical analysis and, due to the linearity of the model, superposition of the elastic state applies:

$$
\langle T\rangle=\frac{1}{V} \int_{V} K\left(D\left(\boldsymbol{u}^{p}\right)+\langle D\rangle\right) d V .
$$

The elements of the homogenized elasticity tensor are calculated separately producing the different elementary strain tensors in the numerical model, by means of (2-7).

A solution of (2-6) by means of FEM analysis can be achieved with the proper boundary conditions on the cell chosen as the representative volume element of the homogenization problem; this choice is crucial. It is well known that several representative volume elements can be chosen to describe a periodic geometry, associated with the appropriate frame of reference and with appropriate integers representing the periods of the geometry [Anthoine 1995]. Usually the smallest one is thought to be the most useful, as the number of unknowns to be determined in the FEM calculation is fewer. On the contrary, as it will be shown, the use of a symmetric RVE may be preferable to a smaller one, because of the ease in imposing boundary conditions [Suquet 1985].

Assume for the periodic solid of Figure 1 a representative volume element characterized by two symmetry planes and an orthogonal frame of reference. Actually, the plane orthogonal to the fiber can be thought of as a symmetry plane as well. The volume subjected to numerical analysis (see Figure 2) is

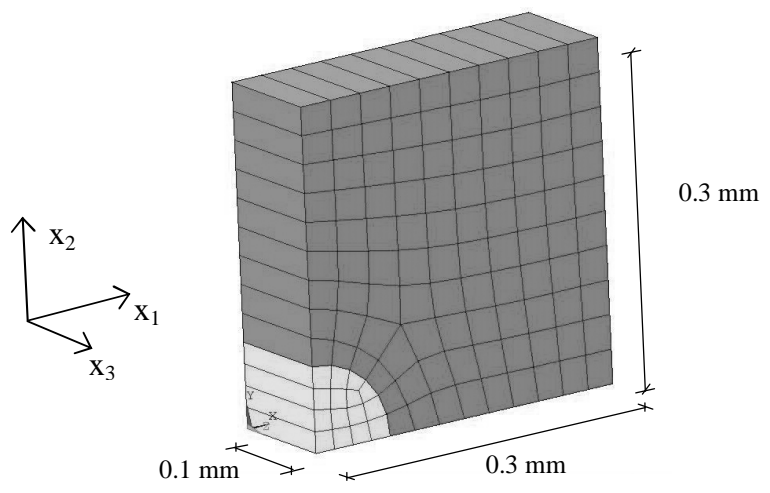

Figure 2. Element subjected to numerical analysis in the case of RVE far from the boundary. 
one-quarter of the representative volume element. In this case, it is evident that the periodicity planes represent, also, symmetry planes with respect to the composite pattern.

The body forces applied to the interface between the two materials and deduced from (2-6) and from the elementary strain states, are in turn symmetric and skew symmetric. In particular, when unit strain tensors $D_{1}, D_{2}$, and $D_{3}$ are applied, the stress vector field in the interface is symmetric, and, due to the symmetry, the constraints prevent the boundary of the RVE from performing displacements normal to the boundary itself. This means that, on the boundary, $\boldsymbol{u}^{p}$ has no components out of the boundary planes. The final deformed RVE will display undistorted planes, which are not deformed with respect to the displacement component orthogonal to the plane itself. Figure 3 shows strain fluctuation, homogeneous strain, and total strain for the RVE chosen for the analysis of the composite under investigation,

When unit strain tensors $D_{4}, D_{5}$, and $D_{6}$ are applied, the stress vector field in the interface is skew symmetric, and, due to the symmetry, the constraints prevent the boundary of the RVE from performing displacements parallel to the boundary itself. This means that, on the boundary, $\boldsymbol{u}^{p}$ has no components on the boundary planes. The final deformed RVE will display planes not deformed with respect to the displacement components parallel to the planes.

According to several authors, the elementary strain can be otherwise imposed directly on the RVE with the so called displacement method [Lukkassen et al. 1995]. In this case the boundary conditions must be compatible with the periodicity but must impose, in turn, the desired boundary displacement; for example the homogeneous strain $D_{1}$ can be directly imposed substituting the boundary condition on the displacement $u_{1}=D_{1} x_{1}$ in place of the homogeneous boundary condition $u_{1}=0$. The other boundary conditions are not as easy to visualize as in the previous case and an accurate analysis of the displacement field and of the algebraic representation of the periodicity conditions is necessary.

As a comparison, the homogenization procedure was performed with the closed-form method described by Zhao and Weng [1990], which makes use of the elastic solution derived by Eshelby [1957] in the case of an infinitely extended matrix containing an ellipsoidal inhomogeneity. Here, the theory of Mori and Tanaka [1973] was not applied to extend Eshelby's solution in order to account for the reciprocal influence of inclusions, since the latter is not significant when the volume fraction is lower than $10 \%$. Eshelby's solution was employed to determine stress and strain localization tensors in the inclusion and the average localization tensors in the matrix.

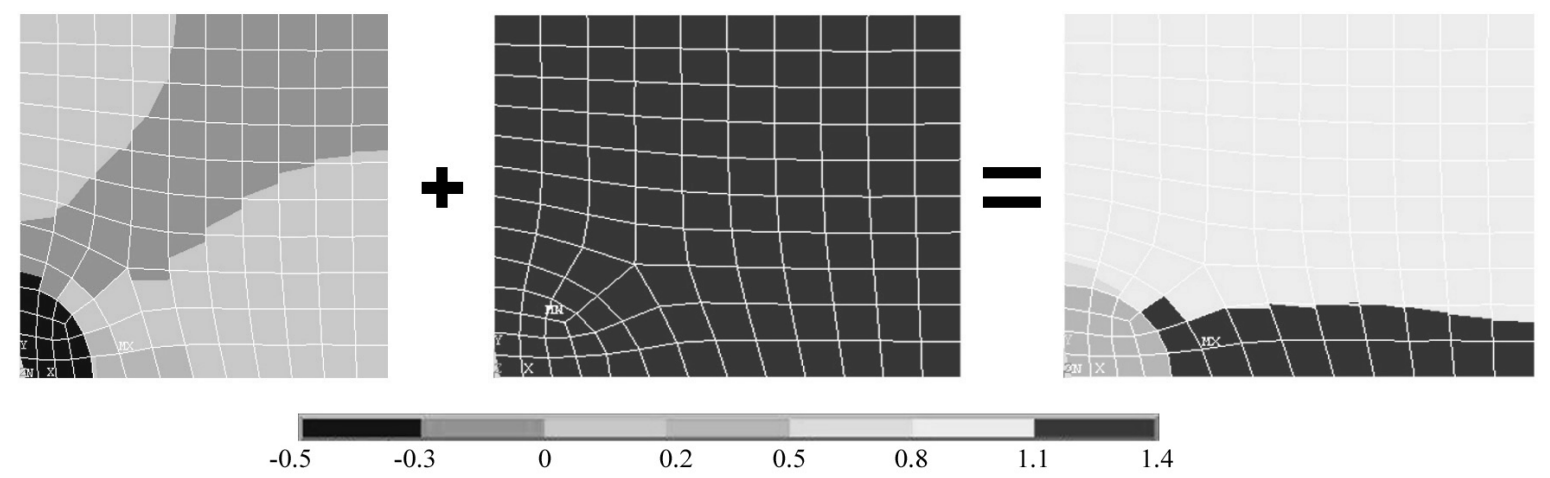

Figure 3. Fluctuation of strain, homogeneous strain, and total strain. 


\begin{tabular}{rcccccc|}
\hline Model & $k_{11}=k_{22}$ & $k_{33}$ & $E_{x}=E_{y}$ & $E_{z}$ & $G_{y z}=G_{z x}$ & $G_{x y}$ \\
Closed-form & 44298.6 & 45558.4 & 21264.1 & 22461.0 & 7717.5 & 7682.5 \\
Numerical RVE far from body boundary & 44320.8 & 45559.1 & 21302.7 & 22461.2 & 7021.2 & 6923.4 \\
Numerical RVE near body boundary & 44612.3 & - & - & - & - & 6927.2 \\
\hline
\end{tabular}

Table 1. Main components of stiffness tensor, Young's modulus, and shear modulus for RVE determined by means of the closed-form procedure and numerical homogenization (in units of MPa).

According to the classical homogenization procedure [Suquet 1985], the homogenized stiffness tensor $K^{\text {hom }}$ is obtained by the average of the stiffness tensors of the phases weighed with the strain localization tensors $B_{i}(\boldsymbol{x}): K^{\text {hom }}=c_{1} K_{1}\left\langle B_{1}(\boldsymbol{x})\right\rangle+c_{2} K_{2}\left\langle B_{2}(\boldsymbol{x})\right\rangle$, where $c_{1}$ and $c_{2}$ are the concentration factors, $K_{1}$ and $K_{2}$ the stiffness tensors, and $B_{1}(x)$ and $B_{2}(x)$ the localization tensors of the two phases.

Table 1 reports the homogenized elastic constants of the composite material determined with the numerical homogenization procedure described, along with those computed by means of the closed-form homogenization procedure.

2.2. Self-strain induced by SMA activation. In order to evaluate the strain state induced by the activation of the SMA wires, it is sufficient to apply on the boundary of the wire within the composite, the surface stress that would have produced the self-strain in the wire out of the composite; this approach follows the one proposed by Eshelby [1957]. In particular, the strain state produced by the activation of the SMA is purely deviatoric and can be represented by a strain tensor of the type

$$
D^{\prime}=r\left[\begin{array}{rrr}
-\frac{1}{2} & 0 & 0 \\
0 & -\frac{1}{2} & 0 \\
0 & 0 & 1
\end{array}\right],
$$

where $r$ represents the axial self-strain capability of the wire. The stress vector field to be applied on the surface of the wire in the composite takes the form

$$
\boldsymbol{f}=\left(K_{2}\right) D^{\prime} \boldsymbol{n} \delta_{\partial S}
$$

where $\boldsymbol{n}$ is the outward unit normal vector. Owing to the geometric symmetry of the cylinder representing the SMA wire, the stress field is symmetric with respect to the planes that define the RVE. For this reason the boundary of the RVE in the final stage will be undistorted, as it is in the case of the homogenization of the strain tensors $D_{1}, D_{2}$, and $D_{3}$. It is necessary to produce a constraint system on the RVE that forces this kind of deformation; this can be achieved by imposing on the boundary of the RVE the same constraint as in the homogenization procedure, that is, preventing the boundary of the RVE from performing displacements normal to the boundary. When this is done, only the fluctuating part of the strain is computed, while the homogeneous part has to be determined subsequently.

The stress state corresponding to the fluctuating part of displacement can be computed from the elastic state obtained via the numerical analysis of the RVE, simply subtracting from the elements constituting the SMA inclusion the stress corresponding to the self-strain, so that equilibrium is reintroduced on the boundary of the SMA wire: 


\begin{tabular}{|rcc|}
\hline Model & $\left\langle\varepsilon_{x}\right\rangle=\left\langle\varepsilon_{y}\right\rangle$ & $\left\langle\varepsilon_{z}\right\rangle$ \\
Numerical RVE far from body boundary & 0.003455 & -0.007591 \\
Numerical RVE near body boundary & 0.001610 & - \\
\hline
\end{tabular}

Table 2. Average strain of the composite material produced by a self-strain $r=5 \%$.

$$
T=-\left(K_{2}\right) D^{\prime} \quad \text { in } V_{2} \text {. }
$$

In order to determine the homogeneous part of the strain field to be superimposed on the fluctuating part determined by the numerical analysis, the condition that the volume average of stress has to be zero in a body subjected to self-strain induced by internal causes is usually introduced:

$$
\langle T\rangle=\sum_{j=1}^{6} \alpha_{j}\left\langle T\left(D_{j}\right)\right\rangle+\int_{\mathrm{RVE}} T^{\mathrm{num}} d V=0 .
$$

This is formally a set of six equations in six unknowns, but the average stresses corresponding to $D_{4}, D_{5}$, and $D_{6}$ vanish because of the symmetry conditions, so the forth, fifth, and sixth equations are identities. The proof of this argument is given in the Appendix.

Table 2 reports the values of the average strain produced in the composite by the activation of the SMA wires, and also, on the last line, the results of the analysis of an RVE supposed to be near to the border of the body. The periodicity conditions are modified, as the $x_{3}$ axis is no longer a direction of periodicity. The analysis has been performed on a RVE long enough to represent, in correspondence with the mean plane, the behavior of the RVE analyzed before and supposed as far from the boundary. Figure 4 reports the tangential stress on transversal cross sections of the RVE in correspondence with a
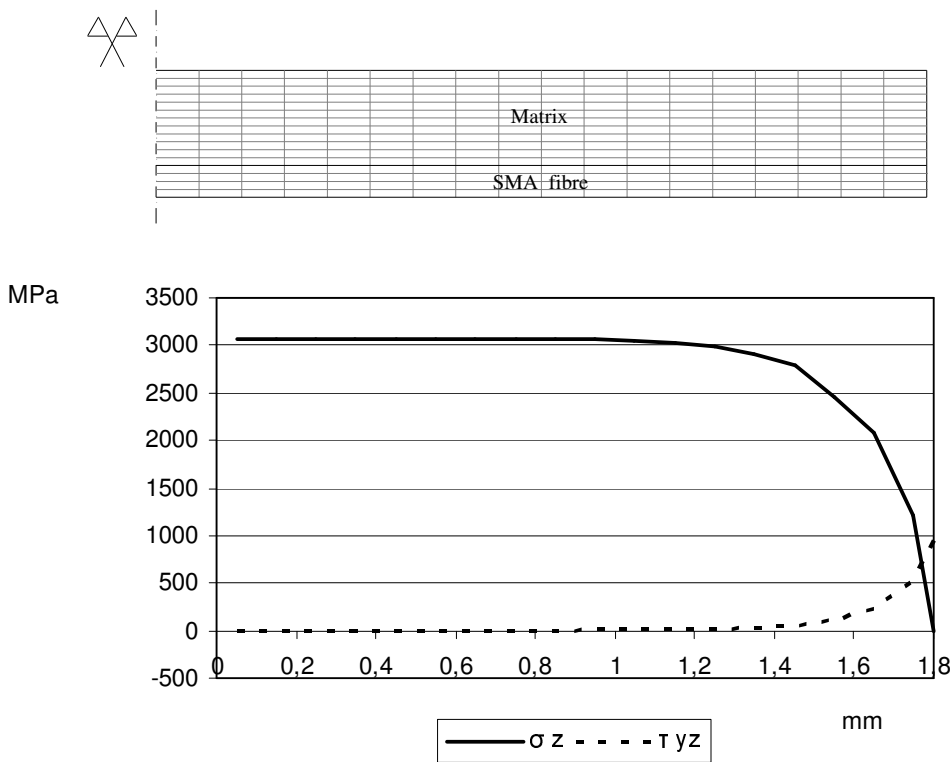

Figure 4. Normal and tangential stress $(\mathrm{MPa})$ in the elements of inclusion corresponding with interface, as a function of the distance from the symmetry plane ( $\mathrm{mm})$. 
progressively larger distance from the boundary of the body. These results can be employed to evaluate the tangential stresses that are generated at the end of the fiber and to check the feasibility of such a composite once the adhesion capability of the two phases is known.

\section{Elastic field in a plate}

In this paper attention is focused on the bending of a plate produced by the self-strain of an embedded SMA wire. The plate is supposed free from constraints and made of a resin characterized by low elastic modulus and high break deformation, which also supports the relatively large strain capability of the SMA wire. The polyester resin Distitron ${ }^{\circledR} 166 \mathrm{UV}$ was selected for the analysis and tested in compression according to [ISO 604 1993]. The experimental campaign carried out on three different polymer materials is described in [Alecci and Ranocchiai 2007].

The eccentricity of the wire with respect to the middle plane of the plate produces the bending of the plate, and is the cause of the coupling of the normal strain and moments, as well as the curvature and normal stress resultants.

3.1. Homogenization. The geometry of the medium is shown in Figure 5. The RVE and the coordinate system are shown in Figure 6. The SMA wire was $500 \mu \mathrm{m}$ in diameter and, as a consequence, the volume fraction of the inclusion is about $2 \%$. The two phases are assumed to be isotropic with the following elastic constants: $E=70000 \mathrm{MPa}$ and $v=0.33$ (fiber), and $E=900 \mathrm{MPa}$ and $v=0.4$ (matrix). The periodicity condition is valid along the $x_{1}$ and $x_{3}$ axes. The linear segments orthogonal to the middle plane are assumed to remain linear after the deformation and rotation is assumed small.

The strain periodic displacement field that respects the periodicity conditions and guarantees the kind of deformation assumed previously is that proposed by Anthoine [1995]:

$$
\begin{aligned}
& u_{1}=d_{11} x_{1}+d_{13} x_{3}+x_{2}\left(\chi_{11} x_{1}+\chi_{13} x_{3}\right)+u_{1}^{\mathrm{per}}, \\
& u_{2}=-\frac{1}{2} \chi_{11} x_{1}^{2}-\frac{1}{2} \chi_{33} x_{3}^{2}-\chi_{13} x_{1} x_{3}+u_{2}^{\mathrm{per}}, \\
& u_{3}=d_{31} x_{1}+d_{33} x_{3}+x_{2}\left(\chi_{31} x_{1}+\chi_{33} x_{3}\right)+u_{3}^{\mathrm{per}} .
\end{aligned}
$$

Note that the coefficients representing the average strain components $\left(d_{11}, d_{13}, \ldots\right)$ have only indices 1 and 3 , as the homogenization procedure according to the periodicity directions is significant; also the coefficients $\chi_{11}, \chi_{13}, \ldots$ have only indices 1 and 3 , as they represent the average curvature components

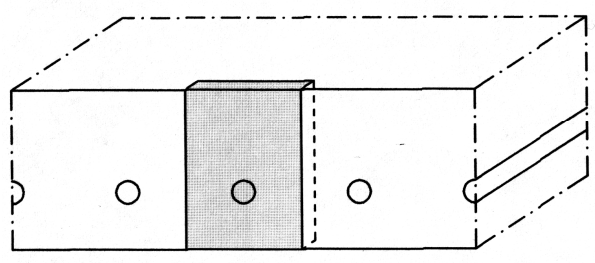

(a)

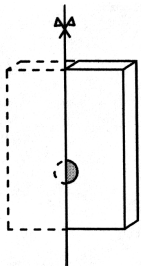

(b)

Figure 5. Left: schematic representation of the composite plate with embedded SMA fibers and localization of the unit cell. The plane shown is perpendicular to the fiber length. Right: region subjected to numerical analysis (continuous line). 


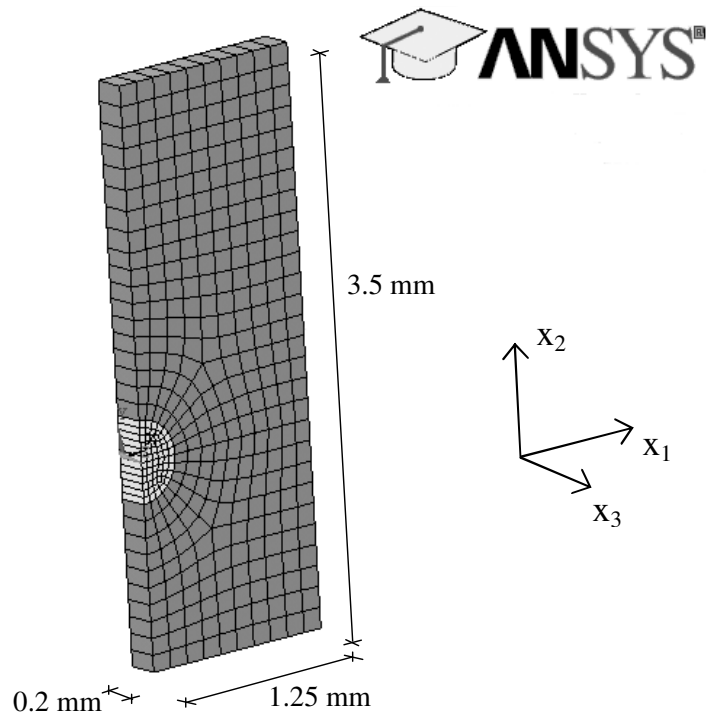

Figure 6. Plate element subjected to numerical analysis.

of the plate having extension in the $x_{1} x_{3}$ plane. Also the periodic displacement components $u_{1}^{\text {per }}, u_{2}^{\text {per }}$, and $u_{3}^{\text {per }}$ depend on the three variables $x_{1}, x_{2}$, and $x_{3}$, but they are periodic only in the axes $x_{1}$ and $x_{3}$.

It is convenient to perform the numerical homogenization in the framework of the displacement method, that is imposing one at a time the homogeneous strain components and average curvature components by means of the corresponding displacements on the boundary, as shown in (3-1) and with the proper boundary conditions. The values of normal stress components and of moment components resulting from the processing of the numerical analysis permits us to deduce the components of the constitutive matrix of the plate:

$$
\left\{\begin{array}{l}
N_{11} \\
N_{33} \\
N_{13} \\
M_{11} \\
M_{33} \\
M_{13}
\end{array}\right\}=\left[\begin{array}{llllll}
A_{11} & A_{13} & A_{14} & B_{11} & B_{13} & B_{14} \\
A_{13} & A_{33} & A_{34} & B_{13} & B_{33} & B_{34} \\
A_{14} & A_{34} & A_{44} & B_{14} & B_{34} & B_{44} \\
B_{11} & B_{13} & B_{14} & D_{11} & D_{13} & D_{14} \\
B_{13} & B_{33} & B_{34} & D_{13} & D_{33} & D_{34} \\
B_{14} & B_{34} & B_{44} & D_{14} & D_{34} & D_{44}
\end{array}\right]\left\{\begin{array}{c}
d_{11} \\
d_{33} \\
2 d_{13} \\
\chi_{11} \\
\chi_{33} \\
2 \chi_{13}
\end{array}\right\} .
$$

The stiffness matrix reported here is often represented partitioned into submatrices $A, B$, and $D$. Its symmetry and the symmetry of the submatrixes are a consequence of the symmetry of the elasticity tensor.

When imposing symmetric strain components, like $d_{11}, d_{33}, \chi_{11}$, and $\chi_{33}$, the displacement components $u_{1}^{\text {per }}$ and $u_{3}^{\text {per }}$ are zero respectively on the planes orthogonal to the $\boldsymbol{n}_{1}$ and $\boldsymbol{n}_{3}$ unit vectors belonging to the boundary of the RVE, representing symmetry planes; this means that the nodes on the boundary cannot move out of the planes. On the contrary, the displacement out of the plane $u_{2}^{\text {per }}$ must be kept free from constraints also on the external boundary of RVE, normal to $\boldsymbol{n}_{2}$. The upper and lower surfaces of the plate have to be left free from constraints. This allows a symmetric deformation of the RVE. 
When imposing skew symmetric strain components, like $d_{13}$ and $\chi_{13}$, the displacement component $u_{1}^{\text {per }}$ is zero on the boundary plane orthogonal to the $\boldsymbol{n}_{3}$ unit vector and $u_{3}^{\text {per }}$ is zero on the boundary plane orthogonal to the $\boldsymbol{n}_{1}$ unit vector. This allows a skew symmetric deformation of the RVE.

For this reason, coefficients $A_{14}, A_{34}, B_{14}, B_{34}, D_{14}$, and $D_{34}$ are zero, as can be deduced observing that, from a macroscopic point of view, the plate is orthotropic and that axes 1 and 3 are orthotropy axes.

The results obtained by the homogenization procedure are reported in Table 3.

3.2. Self-strain induced by SMA activation. According to the method applied in Section 2.2, a surface stress vector field has to be applied on the boundary of the wire within the matrix of the RVE, which would have produced the self-strain if the wire was out of the matrix. The vector field is described in $(2-10)$.

The stress system is again symmetric with respect to planes $x_{1}=0$ and $x_{3}=0$, so that the boundary constraints must be chosen as to prevent antisymmetric displacements. In this case, $u_{1}$ must be zeroed on the boundary planes of periodicity normal to the $\boldsymbol{n}_{1}$ unit vector, and $u_{3}$ must be zeroed on planes of periodicity normal to the $\boldsymbol{n}_{3}$ unit vector. Vertical displacements $u_{2}$ are allowed by the requirement for symmetry.

As in the previous case, the analysis reproduces the fluctuating part of strain, while the stress state can be computed by subtracting the stress corresponding with the self-strain from the elements constituting the SMA inclusion, according to (2-11), so that the equilibrium is reintroduced on the boundary of the SMA wire.

In order to determine the homogeneous part of the strain field to be superimposed on the fluctuating part determined by the numerical analysis, the condition that the volume average of the stress has to be zero in a body subjected to self-strain induced by internal causes is introduced. This time the plate normal stress and moments have to be zeroed by adding the plate strain components able to produce the opposite values of internal force; these can be computed by knowing the plate stiffness of (3-2) reported in Table 3.

Average strain and curvature produced by a self-strain of $r=3 \%$ are reported in Table 4 . The stress distribution on the plane $x_{3}=0$ is reported in Figure 7.

\begin{tabular}{|cccc|}
\hline$A_{11}(\mathrm{~N} / \mathrm{mm})$ & $A_{13}(\mathrm{~N} / \mathrm{mm})$ & $A_{33}(\mathrm{~N} / \mathrm{mm})$ & $A_{44}(\mathrm{~N} / \mathrm{mm})$ \\
3891.75 & 1549.29 & 9159.54 & 2335.13 \\
\hline$B_{11}(\mathrm{~N})$ & $B_{13}(\mathrm{~N})$ & $B_{33}(\mathrm{~N})$ & $B_{44}(\mathrm{~N})$ \\
70.85 & 24.65 & 2704.77 & 42.56 \\
\hline$D_{11}(\mathrm{~N} \mathrm{~mm})$ & $D_{13}(\mathrm{~N} \mathrm{~mm})$ & $D_{33}(\mathrm{~N} \mathrm{~mm})$ & $D_{44}(\mathrm{~N} \mathrm{~mm})$ \\
3866.28 & 1544.43 & 5264.49 & 2326 \\
\hline
\end{tabular}

Table 3. Components of the constitutive matrix of the plate.

\begin{tabular}{|cccc|}
\hline$\left\langle d_{11}\right\rangle$ & $\left\langle d_{33}\right\rangle$ & $\left\langle\chi_{11}\right\rangle$ & $\left\langle\chi_{33}\right\rangle$ \\
0.006231 & -0.016608 & 0.003022 & -0.007949 \\
\hline
\end{tabular}

Table 4. Average strain and curvature produced by a self-strain of $r=3 \%$. 

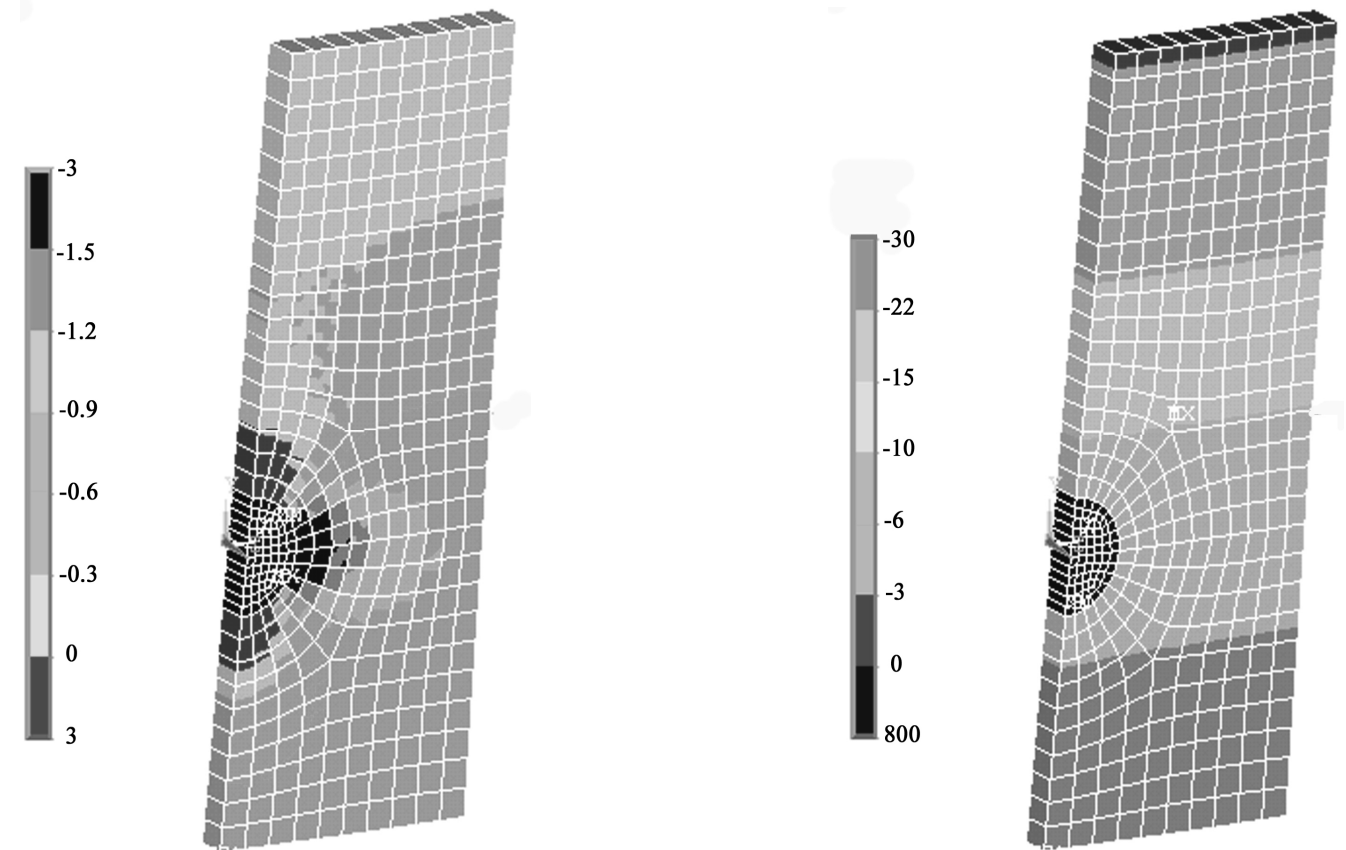

Figure 7. Normal stress $(\mathrm{MPa}) \sigma_{x}$ (left) and $\sigma_{z}$ (right) in the plate element subjected to numerical analysis.

We note that the curvature tensor is negative definite at every point of the mean surface of the RVE, which is then a hyperbolic surface.

\section{Conclusions}

Numerical procedures have been described for the homogenization and the evaluation of the stress field in a composite as consequence of the shape-memory effect of embedded SMA wires. Such procedures proved suitable for the study of self-strain induced by SMA activation. In particular, it was shown that the individuation of the boundary conditions is strictly dependent on the choice of the RVE, and that it is simpler if the boundary constraints are individuated when the boundary of the RVE is also a symmetry plane for the composite.

As was expected, the stress state determined in the RVE, at the end of the SMA wire activation process, far from boundary is quite similar to the stress state in the symmetry plane of the RVE located near to the boundary. Nevertheless, this last case analysis is useful in order to evaluate the tangential stresses that are generated at the end of the fiber, the first step for the design of a real composite, once the adhesion properties of the constituents are experimentally investigated.

The analysis of a composite plate can produce the homogenized stiffness matrix of the plate, and the average main curvatures and strain of the composite plate, under the small rotation assumption. 


\section{Appendix: Derivation of the null average stress condition}

The assumption of null average stress relies on the theory of dislocation. Volterra, in 1907, first used the term "distorsione", which Love [1927] translated as "dislocation"; nowadays the word dislocation is mainly used to indicate the defects of crystal lattices and the theory of dislocation explains the plasticity and work hardening of crystals.

When a body is not subjected to external forces, that is load and constraint forces, an eigenstrain produces an internal stress field. The body is free from constraints or it is constrained such as to be an isostatic system. This is the case of pretensioned, prestressed, reinforced concrete beams. The condition that average stress is null in the pretensioned, prestressed beam sections is usually employed and is a special case of (2-12), following simple equilibrium considerations. Equation (2-12) acquires particular interest when dealing with homogenization problems, because average stress is one of the basic quantities used in homogenization theory. Equation (2-12) can be easily proven by means of Signorini's theorem [Gurtin 1981].

In the case of no body forces acting on a finite region $\Omega$ and supposing that $T$ is smooth, Signorini's theorem states that:

$$
V(\Omega)\langle T\rangle=\int_{\partial \Omega}(T n \otimes r) d A,
$$

where $V(\Omega)$ is the volume of the body, $T$ the stress tensor, $n$ the unit vector normal to the boundary $\partial \Omega$, and $r$ the position vector. If a portion $\Omega_{1}$ of a body is subjected to an eigenstrain (see Figure 8), a surface stress state develops, opposite and equal on the two opposite sides of the boundary between $\Omega_{1}$ and $\Omega_{2}$, being $\Omega_{2}$ its complementary part. We can write Signorini's formula for the two regions:

$$
V\left(\Omega_{1}\right)\left\langle T_{1}\right\rangle=\int_{\partial \Omega_{1}}\left(T_{1} n_{1} \otimes r\right) d A, \quad V\left(\Omega_{2}\right)\left\langle T_{2}\right\rangle=\int_{\partial \Omega_{2}}\left(T_{2} n_{2} \otimes r\right) d A .
$$

Since $n_{1}=-n_{2}, \partial \Omega_{1}=\partial \Omega_{2}$, and $T_{1} n_{1}=-T_{2} n_{2}$ (from the equilibrium condition), we have

$$
V\left(\Omega_{1}\right)\left\langle T_{1}\right\rangle=\int_{\partial \Omega_{1}}\left(T_{1} n_{1} \otimes r\right) d A=\int_{\partial \Omega_{2}}-\left(T_{2} n_{2} \otimes r\right) d A=-V\left(\Omega_{2}\right)\left\langle T_{2}\right\rangle,
$$

that is

$$
V\left(\Omega_{1}\right)\left\langle T_{1}\right\rangle+V\left(\Omega_{2}\right)\left\langle T_{2}\right\rangle=0
$$

which is equivalent to (2-12).
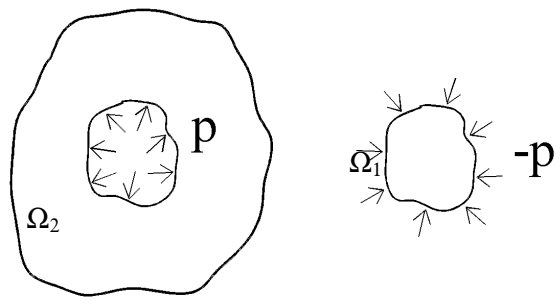

Figure 8. A region subjected to eigenstrain produces interfacial stress vector. 


\section{References}

[Aboudi 1997] J. Aboudi, “The response of shape memory alloy composites”, Smart Mater. Struct. 6:1 (1997), 1-9.

[Ahmad et al. 1990] I. Ahmad, A. Crowson, C. A. Rogers, and M. Aizawa (editors), US-Japan Workshop on Smart/Intelligent Materials and Systems (Honolulu, HI, 1990), Technomic, Lancaster, PA, 1990.

[Alecci and Ranocchiai 2007] V. Alecci and G. Ranocchiai, "Caratterizzazione meccanica di materiali polimerici per la realizzazione di un composito attivo", pp. 782-791 in Sperimentazione su materiali e strutture: convegno nazionale (Venice, 2006), edited by S. Russo and E. Siviero, Università IUAV di Venezia, DCA, LabSCo, 2007.

[Anthoine 1995] A. Anthoine, "Derivation of the in-plane elastic characteristics of masonry through homogenization theory", Int. J. Solids Struct. 32:2 (1995), 137-163.

[Anthoine 1997] A. Anthoine, "Homogenization of periodic masonry: plane stress, generalized plane strain or 3D modelling?", Commun. Numer. Methods Eng. 13:5 (1997), 319-326.

[Baltá et al. 2002] J. A. Baltá, V. Michaud, J. Månson, M. Parlinska, and R. Gotthardt, "Adaptive composite materials processing", pp. 1-6 in Composites for the future (ECCM-10) (Brugge, 2002), edited by H. Sol and J. Degrieck, ESCM, Brugge, 2002 .

[Barrett and Gross 1996] R. Barrett and R. S. Gross, "Super-active shape-memory alloy composites", Smart Mater. Struct. 5:3 (1996), 255-260.

[Bidaux et al. 1994] J. E. Bidaux, J. A. E. Manson, and R. Gotthardt, "Dynamic mechanical behavior and phase transformation of polymer based shape memory alloys composites", pp. 37-42 in Proceedings of SMST-94: the First International Conference on Shape Memory and Superelastic Technologies (Pacific Grove, CA, 1994), edited by A. R. Pelton et al., Monterey Institute for Advanced Studies, Monterey, CA, 1994.

[Boyd and Lagoudas 1994] J. G. Boyd and D. C. Lagoudas, "Thermomechanical response of shape memory composites", J. Intell. Mater. Syst. Struct. 5:3 (1994), 333-346.

[Cecchi et al. 2005] A. Cecchi, G. Milani, and A. Tralli, "Validation of analytical multiparameter homogenization models for out-of-plane loaded masonry walls by means of the finite element method", J. Eng. Mech. (ASCE) 131:2 (2005), 185-198.

[Daghia et al. 2006] F. Daghia, F. Ubertini, E. Viola, and D. J. Inman, "A finite element model for SMA hybrid composite plates", in Atti del XVI Convegno Italiano di Meccanica Computazionale (Bologna, 2006), edited by F. Ubertini et al., Officine Grafiche Tecnoprint, Bologna, 2006.

[Duvaut 1984] C. Duvaut, "Homogénéisation et matériaux composites", pp. 35-62 in Trends and applications of pure mathematics to mechanics (Palaiseau, 1983), edited by P. G. Ciarlet and M. Roseau, Lecture Notes in Physics 195, Springer, Berlin, 1984.

[Eshelby 1957] J. D. Eshelby, “The determination of the elastic field of an ellipsoidal inclusion, and related problems”, Proc. R. Soc. Lond. A 241:1226 (1957), 376-396.

[Gurtin 1981] M. E. Gurtin, An introduction to continuum mechanics, Academic Press, Boston, 1981.

[Hecker et al. 1970] S. S. Hecker, C. H. Hamilton, and L. J. Ebert, "Elastoplastic analysis of residual stresses and axial loading in composite cylinders", J. Mater. 5:4 (1970), 868-900.

[ISO 604 1993] ISO 604, "Plastics: determination of compressive properties", 1993. Superseded by ISO 604:2002.

[Love 1927] A. E. H. Love, A treatise on the mathematical theory of elasticity, 4th ed., Cambridge Univ. Press, Cambridge, 1927.

[Lukkassen et al. 1995] D. Lukkassen, L.-E. Persson, and P. Wall, "Some engineering and mathematical aspects on the homogenization method", Compos. Eng. 5:5 (1995), 519-531.

[Marfia 2005] S. Marfia, "Micro-macro analysis of shape memory alloy composites", Int. J. Solids Struct. 42:13 (2005), 36773699.

[Marfia and Sacco 2005] S. Marfia and E. Sacco, "Micromechanics and homogenization of SMA-wire-reinforced materials", J. Appl. Mech. (ASME) 72:2 (2005), 259-268.

[Marfia and Sacco 2007] S. Marfia and E. Sacco, "Analysis of SMA composite laminates using a multiscale modelling technique”, Int. J. Numer. Methods Eng. 70:10 (2007), 1182-1208. 
[Milani et al. 2006] G. Milani, P. Laurenço, and A. Tralli, "Homogenization approach for the limit analysis of out-of-plane loaded masonry walls", J. Struct. Eng. (ASCE) 132:10 (2006), 1650-1663.

[Mori and Tanaka 1973] T. Mori and K. Tanaka, "Average stress in matrix and average elastic energy of materials with misfitting inclusions", Acta Metall. 21:5 (1973), 571-574.

[Murasawa et al. 2004] G. Murasawa, K. Tohgo, and H. Ishii, "Deformation behavior of NiTi/polymer shape memory alloy composites: experimental verifications", J. Compos. Mater. 38:5 (2004), 399-416.

[Suquet 1985] P. M. Suquet, "Elements of homogenization for inelastic solid machanics", pp. 194-230 in Homogenization techniques for composite media, edited by E. Sanchez-Palencia and A. Zaoui, Springer, Berlin, 1985.

[Tsoi et al. 2004] K. A. Tsoi, J. Schrooten, Y. Zheng, and R. Stalmans, "II: Thermomechanical characteristics of shape memory alloy composites", Mater. Sci. Eng. A 368:1-2 (2004), 299-310.

[Wei et al. 1997] Z. G. Wei, C. Y. Tang, and W. B. Lee, "Design and fabrication of intelligent composites based on shape memory alloys", J. Mater. Process. Technol. 69:1-3 (1997), 68-74.

[Xu et al. 2002] Y. Xu, K. Otsuka, H. Yoshida, H. Nagai, R. Oishi, H. Horikawa, and T. Kishi, "A new method for fabricating SMA/CFRP smart hybrid composites", Intermetallics 10:4 (2002), 361-369.

[Zhao and Weng 1990] Y. H. Zhao and G. J. Weng, "Effective elastic moduli of ribbon-reinforced composites", J. Appl. Mech. (ASME) 57:1 (1990), 158-166.

[Zheng et al. 2001] Y. Zheng, L. Cui, Y. Li, and R. Stalmans, "Partial transformation behavior of prestrained TiNi fibers in composites", Mater. Lett. 51:5 (2001), 425-428.

[Zheng et al. 2002] Y. J. Zheng, J. Schrooten, K. A. Tsoi, and R. Stalmans, "Thermal response of glass fibre/epoxy composites with embedded TiNiCu alloy wires", Mater. Sci. Eng. A 335:1-2 (2002), 157-163.

[Zheng et al. 2003] Y. J. Zheng, J. Schrooten, K. A. Tsoi, and P. Šittner, "Qualitative and quantitative evaluation of the interface in activated shape memory alloy composites", Exp. Mech. 43:2 (2003), 194-200.

Received 24 Sep 2008. Revised 29 Jun 2009. Accepted 4 Jul 2009.

VALERIO ALECCI: valerio.alecci@unifi.it

Dipartimento di Costruzioni, Università di Firenze, Piazza Brunelleschi 6, 50121 Firenze, Italy

SILVIA BRICCOLI BATI: silvia.briccolibati@unifi.it

Dipartimento di Costruzioni, Università di Firenze, Piazza Brunelleschi 6, 50121 Firenze, Italy

GIOVANNA RANOCCHIAI: giovanna.ranocchiai@unifi.it

Dipartimento di Costruzioni, Università di Firenze, Piazza Brunelleschi 6, 50121 Firenze, Italy 